\title{
TNM8 BREAST CANCER CALCULATOR: A TOOL FOR BREAST CANCER STAGING ACCORDING TO THE TNM $8^{\text {TH }}$ EDITION
}

Wesley Рereira Andrade ${ }^{1}$

${ }^{1}$ Instituto de OncoMastologia - São Paulo (SP), Brazil.

Objectives: To develop an APP to help healthcare professionals around the world in the breast cancer staging process according to the new AJCC criteria. Methodology: Extensive revision of the AJCC Cancer Staging Manual 8th Edition and the Updated Breast Chapter with a high improvement of the knowledge in this field. Creation of the medical interface for computer interface. Results: Development of the software named TNM8 Breast Cancer Calculator that is an updated, complete, intuitive, and user-friendly tool for Breast Cancer Staging. All data and definitions are based on the Updated Breast Chapter - AJCC Cancer Staging Manual 8th Edition, released on November 10th, 2017 that included the classic Anatomic Stage Group and the new and revolutionary Prognostic Stage Group (divided in Clinical Prognostic Stage and Pathological Prognostic Stage) with the incorporation of biomarker factors. The prognostic factors included are: Tumor Grade (G), HER2, Estrogen Receptor (ER) and Progesterone Receptor (PR). Genomic Profiles were also incorporated as a prognostic factor for eligible cases (pT1-T2 N0 M0 HER2- and ER+) in the Pathological Prognostic Stage. This App provides the quantification of the category change of the Clinical Prognosis Stage (cPS) and also the Pathological Prognostic Stage (pPS) in relation to the Anatomic Stage (AS) expressed in the Staging Line with the symbol $+1,+2,+3$ in case of worsening in the Prognostic Staging or -1, $-2,-3$ in case of improvement in the Prognostic Staging. When no symbol is shown, it is because the AS and cPS or AS and pPS are identical. This App has other function that shows Oncotype DX ${ }^{\circledR}$ / Genomic Profile field only in some setting when the genomic test is potentially indicated (pT1-T2 N0, HER2- and ER+). The TNM8 Breast Cancer Calculator got the approval of the AJCC and American College of Surgeons. Conclusion: The new TNM for breast cancer changed the process of breast cancer staging. These updates will provide additional precision and flexibility to the staging system but with a complex process. For a correct staging process, doctors need to use complex tables or can use the smart tools to help them in this process. TNM8 Breast Cancer Calculator is very useful for breast surgeons, surgical oncologists, medical oncologists, radiation oncologists, pathologists, radiologists, medical students, scientists, researchers, and all healthcare professionals working in the field of breast oncology. 